\title{
Reliable Positioning with Hybrid Antenna Model for Aerial Wireless Sensor and Actor Networks
}

\author{
Kai Li*, Mustafa İlhan Akbaş ${ }^{\dagger}$, Damla Turgut ${ }^{\dagger}$, Salil S. Kanhere* and Sanjay Jha* \\ * School of Computer Science and Engineering \\ The University of New South Wales, Sydney, Australia \\ Email: \{kail,salilk,sanjay\}@ cse.unsw.edu.au \\ ${ }^{\dagger}$ Department of Electrical Engineering and Computer Science \\ University of Central Florida, Orlando FL \\ Email: \{miakbas,turgut $\} @$ eecs.ucf.edu
}

\begin{abstract}
Aerial wireless sensor and actor networks are composed of multiple unmanned aerial vehicles. An actor node in the network has the capabilities of both acting on the environment and also performing networking functionalities for sensor nodes. Thus, positioning of actors is critical for the efficient data collection. In this paper, we propose an actor positioning strategy, which utilizes a hybrid antenna model that combines the complimentary features of an isotropic omni radio and directional antennas. We present a distributed algorithm for fast neighbor discovery with the hybrid antenna. The omni module of the hybrid antenna is used to form a self organizing network and the directional module is used for reliable data transmission. Extensive simulations show that our protocol improves the packet reception ratio by up to $50 \%$ compared to omnidirectional antenna. Moreover, the network reorganization delay is also reduced. The tradeoff between coverage and reorganization delay is also illustrated.
\end{abstract}

\section{INTRODUCTION}

Wireless Sensor and Actor Networks (WSANs) [1] consist of a large number of sensor nodes with limited capabilities, and a smaller number of actors, which process the collected information and react accordingly. The recent advances in Unmanned Aerial Vehicles (UAVs) made it possible to deploy Aerial Wireless Sensor and Actor Networks (AWSANs). UAVs have been equipped with various types of sensors such as visual [2] or thermal [3], for collecting environmental data, which increases their popularity in a wide range of applications [4].

In AWSANs, actors act on the environment by using actuators such as servo-mechanisms. For instance, lowflying helicopter platform proposed by Thrun et al. [5] provides ground mapping and air-to-ground cooperation for autonomous robotic vehicles. Besides acting on the environment and collecting data, actors also perform networking-related functionalities such as processing or relaying data in multiUAV systems. Compared to single-UAV systems, multi-UAV systems have advantages such as scalability and survivability. There are numerous applications for multi-UAV systems and some of these applications such as toxic plume observation or atmospheric sensing focus on continuous observation of three dimensional (3-D) space.

The formation and dynamic adaptation of the network topology in 3-D space is important for the coverage of the 3-D environment and the effective data collection. Sensor networks have been utilized recently for applications in 3D space such as space exploration, airborne and underwater surveillance [4]. However, these solutions in different domains do not directly apply to UAV systems, which have their own unique challenges. For instance, the dynamic environmental conditions and node movements complicate the maintenance of communication links. The nodes in AWSANs are mobile with higher speeds compared to most other systems. Therefore, the topology changes are more frequent. The radio links and physical layer characteristics are also different in AWSANs since the communication ranges are generally longer than underwater sensor networks or vehicular networks. Thus, a positioning strategy for AWSANs must be able to attain and maintain 3-D coverage of the observed environment while taking these constraints into account.

In our initial work ([6], [7]) a scalable algorithm for dynamic positioning of actors is proposed to provide high connectivity and coverage. In this paper, we apply our novel actor positioning strategy to real-world scenarios by utilizing a rotatable hybrid antenna model (O-BESPAR) [8]. Our positioning algorithm is based on the Valence Shell Electron Pair Repulsion (VSEPR) theory [9], which is utilized to define the positions of actors with respect to the sink. The positioning strategy is adapted to the constraints of UAV systems such as quick neighbor discovery during flight in 3-D space, varying signal strength depending on the antenna orientation and reorganization requirements in case of topology changes. For this purpose, the characteristics of different antenna modules are analyzed based on field experiments. According to results of these experiments, the rotatable hybrid antenna model is utilized with actor-sink communication, actor rearrangement algorithms, and beamforming.

The remainder of the paper is organized as follows. Section II discusses literature review. Section III presents the positioning system model, rotatable O-BESPAR antenna model and communication protocols. The simulation results presented in Section IV demonstrate how our antenna model in conjunction with the positioning algorithm improves the performance of AWSAN. Finally, we conclude in Section V. 


\section{RELATED WORK}

The literature on dynamic node positioning is mostly limited to two dimensional (2-D) space and the designed accordingly become NP-hard in 3-D. 3-D solutions aim to have the least number of polyhedrons for maximal coverage [10]. These assumptions are not applicable in real world environmental monitoring scenarios because of two main challenges. First, the number of nodes and their locations are restricted by the investigated environment and reception ranges of nodes. Second, the dynamic UAV network topology and flight must be handled efficiently considering the properties of the antennas.

Bai et al. [11] studied the problem of constructing a 3-D WSN, which achieves low-connectivity and full-coverage by using the least number of sensors. The 'low connectivity' in this approach is defined as having at least $k$ disjoint paths between any two sensors, where $k \leq 4$. Slab Routing by Chiang and Peng [12] adapts 2-D geographic face routing techniques to 3-D space by dynamically creating a space partition and executing face routing over the planar projected graph of nodes. Lee et al. [13] address the deployment problem for a swarm of autonomous mobile robots. Each robot interacts with three neighboring robots in a selective and dynamic fashion without using any explicit communication so that four robots eventually form a regular tetrahedron. Our approach utilizes VSEPR theory of chemistry, proposed by Gillespie and Nyholm [9]. According to VSEPR theory, the maximum repulsion of the electron pairs or atoms defines the geometric optimum positions of peripheral atoms.

The communication reliability advantages of directional antenna have been discussed in literature (see [14]). Jiang et al. [15] demonstrate a localization scheme using beacon nodes with directional antennas, which rotate regularly. After evaluating the received signal strength indication (RSSI) values of the beacon signals, a sensor node estimates the orientation relative to the beacon node. However, this approach works for the 2-D static or mobile sensor networks but not for 3-D space.

Beam steering based [16] and scan-based [17] algorithms with directional antenna are also proposed. Beam steering works for infrastructure based networks. It is not practical for UAV networks while scan-based approach requires all nodes to follow the same search sequence. Adaptive Medium Access Control protocol for UAV (AMAC_UAV) [18] is designed for a network of UAVs with directional antennas. Each UAV is equipped with two directional antennas for data transmission and two omnidirectional antennas for location packets.

Data transmission via omni antenna is limited by the transmission range of the antenna. As the number of antennas increases, the complexity of the algorithm and the power requirements also increase. Moreover, the data transmitted by omni antenna has a high probability of packet loss, especially when the node is mobile.

\section{Flight And Antenna Model}

\section{A. Aerial positioning model}

The positioning model is designed for determining the locations of actors around a central sink node. The sink
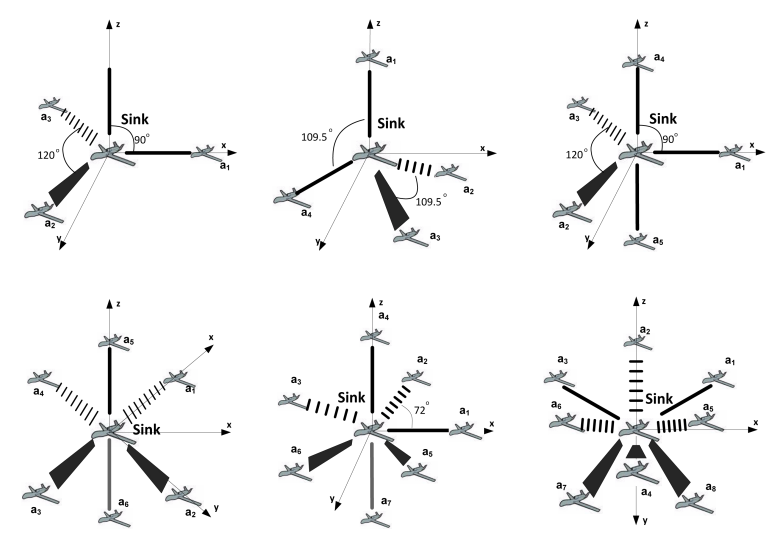

Fig. 1. VSEPR theory geometries for three to eight actors.

node has the highest communication capabilities and it is the main data collector. Actors transmit the collected information to the sink and react to the environmental events according to the application's scope. The network among actors and the sink is the communication backbone of the AWSAN. The positioning strategy preserves 1-hop connectivity between each actor and the sink by using the VSEPR theory, which is the most successful model for prediction of closed-shell molecule geometries. In our approach, the possible actor positions for different number of actors are determined according to VSEPR theory geometries, which are given in Fig. 1. Then these physical locations are converted into a virtual coordinate system with the sink serving as the origin. The number of actors $(n)$ and the communication ranges of the nodes are the defining factors for the locations.

The geometries formed by the UAVs using the VSEPR theory are identified by creating a virtual coordinate system, which has the sink at the origin. The main direction in the flight plan of sink forms the $x$-coordinate and the positions of the actors are defined with respect to the sink and its flight direction. The formulation of geometries is important for the definition of actor positions and the transitions between geometries. The actor positions according to the defined coordinate system are given in Table I. According to VSEPR theory, the actors are located on the $z=0$ plane when the number of actors are less than four. The actors form "Linear" and "Trigonal planar" geometries, in which the angle between two neighboring actors is defined as $\Theta=\frac{360}{n}$. When there are four actors, the sink is located at the center with substituents located at the corners of a tetrahedron with connection angles of $\cos ^{-1}\left(\frac{-1}{3}\right) \approx 109.5^{\circ}$.

The positions of the nodes in VSEPR geometries are calculated such that the least distance between any two pairs of nodes is maximized. To achieve this maximum distance when the number of actors exceeds four, two of the actors are positioned on $z$-axis and the rest of the actors are positioned on the $z=0$ plane with a connection angle of $\Theta=\frac{360}{n-2}$. Hence, "Tetrahedron", "Trigonal bipyramid" and "Octahedron" geometries are formed by five, six and seven actors. When there are eight surrounding actors, the characteristics of VSEPR theory allow multiple possible geometries. However, the square antiprism 
is the favored geometry, which corresponds to the shape when eight points are distributed on the surface of a sphere according to the repulsion among each other. The resulting positions of actors for each geometry are given in Table I.

The positioning algorithm for actors, which uses the number $(n)$ and reception range of actors $(r)$, is given in Algorithm 1. Our positioning approach is improved by using a realistic antenna model and by modifying the positioning algorithm accordingly. Algorithm 1 assumes spherical transmission and reception ranges with identical RSSI and loss rates at every communication angle. However, these factors are effective in the performance of real life UAV systems. Therefore, the characteristics of the antennas are critical to adapt the algorithm to real-life constraints.

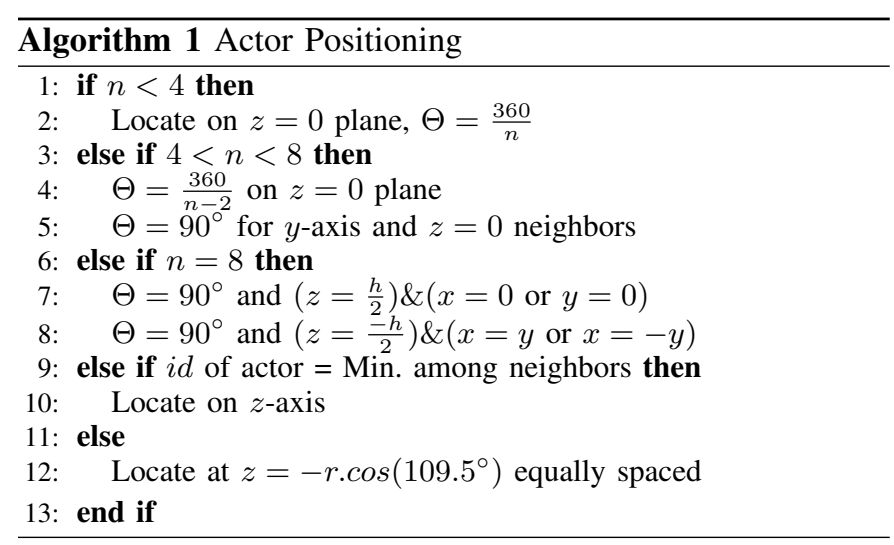

\section{B. O-BESPAR antenna model}

O-BESPAR antenna model leverages the complementary properties of omnidirectional and directional antennas. While omnidirectional antennas enable 360 degrees of coverage when needed, the directional antennas provide high throughput and low interference. In addition to this important unifying characteristic, O-BESPAR is utilized for our flight model based on its several other properties, which can be summarized as follows:

- Utilization of two independent directional beams permits a node to transmit and receive simultaneously.

- The light weight and small size of the module make it rotatable so that the beamforming can be steered to any direction in 3-D.

- Cooperation of omnidirectional and directional antennas permits different transmission rates for data transferring.

Our communication protocol incorporates an efficient neighbor discovery mechanism, which not only allows UAVs to discover each other rapidly but also enables quick alignment of directional beams to maximize the data transfer opportunities. According to our communication protocol, actors broadcast control messages through the omni module in order to exchange location information with the sink. After both beams are steered to each other, the data transmission commences over the directional module. However, the transmission range of the omni antenna is much smaller than the directional ESPAR module. Therefore if no neighbor is found by the broadcast of the omni module, the communication protocol uses the directional module to perform bi-directional beam sweeping. Each beam covers 180 degrees so that the scanning delay is minimized.

GPS receiver and altitude sensor are commonly used builton equipments for UAVs nowadays. After using omnidirectional antenna module to locate the actors, the sink has to calculate the angle difference to steer one of its directional beams to the sink. The angle, $(\phi, \theta)_{A, S}$, between the sink, $S$, and an actor, $A$, is calculated as follows:

$$
(\phi, \theta)_{A, S}=\arctan \left(\frac{z_{A}-z_{S}}{\sqrt{\left(x_{A}-x_{S}\right)^{2}+\left(y_{A}-y_{S}\right)^{2}}}\right)
$$

where $\phi$ and $\theta$ stand for the angle of beamforming in horizontal and vertical plane separately.

We utilize two-ray ground path loss as the propagation model. According to this model, the receiving power $P_{r}$ depends on the transmission power $P_{t}$, antenna gain of transmitter $G_{t}$, antenna gain of receiver $G_{r}$, distance between the actor and sink $d_{A, S}$, the wavelength $\lambda$ and the antenna heights $H_{t}$ and $H_{r}$. The calculation of the receiving power $P_{r}$ is given as follows:

$$
P_{r}=\frac{P_{t} G_{t} G_{r} \lambda^{2}}{(4 \pi d)^{2}}\left[4 \sin \left(\frac{\pi H_{t} H_{r}}{\lambda d}\right)\right]^{2}
$$

In our prior experiments [19], we have shown that the orientation of omni antenna affects the RSSI value. Thus, the omnidirectional antenna is not completely isotropic in a 3-D network. In particular, the measurement of RSSI is extremely important for the positioning schemes for two reasons. First, the neighbor discovery packets are sent from the omni antenna module. If the RSSI value is too low, actor UAV has to sacrifice the coverage to fly closer to the sink. Second, the actor UAVs fly around the sink and keep their positions in the flight. If the sink UAV does not receive the beacon messages from some actor for time out, that actor UAV is assumed to be lost and the network topology is changed to a non optimal geometry.

\section{Communication and rearrangement protocols}

We propose two protocols for the communication between the sink and actor UAVs. There are three main features for these protocols. First, the control packets (10 bytes beacon and 2 bytes ACK) and data packets are transmitted by the omni and directional module separately. Thus, omni module performs fast neighbor discovery and directional module guarantees successful data delivery. Second, the protocol works with our actor positioning algorithm to form and change the VSEPR geometries. In other words, the sink achieves dynamic positioning for the actors. Third, during the flight, the sink must respond to the change of VSEPR geometry efficiently. Since some actors may leave or join the network, the protocol includes a repositioning mechanism which rearranges the actors and updates the beamforming direction.

The main algorithm for the communication between actor and sink UAVs is presented in Algorithm 2. Before transmitting the collected data to the sink, an actor node uses omni antenna module to broadcast beacon messages, which contain 
TABLE I

UAV POSITIONS FOR VSEPR GEOMETRIES

\begin{tabular}{|l|l|}
\hline Geometry & Actor positions \\
\hline Linear & $p_{a_{1}}(x, y, z)=(r, 0,0), p_{a_{2}}(x, y, z)=(-r, 0,0)$ \\
\hline Trigonal planar & $p_{a_{1}}(x, y, z)=(r, 0,0), p_{a_{2}}(x, y, z)=\left(-r \cdot \sin \left(30^{\circ}\right), r \cdot \sin \left(60^{\circ}\right), 0\right), p_{a_{3}}(x, y, z)=\left(-r \cdot \sin \left(30^{\circ}\right),-r \cdot \sin \left(60^{\circ}\right), 0\right)$ \\
\hline Tetrahedron & $p_{a_{1}}(x, y, z)=(0,0, r), p_{a_{2}}(x, y, z)=\left(-r \cdot a,-r \cdot b, r \cdot \cos \left(109 \cdot 5^{\circ}\right)\right)$ \\
& $p_{a_{3}}(x, y, z)=\left(-r \cdot \sin \left(109 \cdot 5^{\circ}\right), 0, r \cdot \cos \left(109 \cdot 5^{\circ}\right)\right), p_{a_{4}}(x, y, z)=\left(-r \cdot a, r \cdot b, r \cdot \cos \left(109 \cdot 5^{\circ}\right)\right)$ \\
& $\left(a=\sin \left(109 \cdot 5^{\circ}\right) \cdot \sin \left(30^{\circ}\right), b=\sin \left(109.5^{\circ}\right) \cdot \cos \left(30^{\circ}\right)\right)$ \\
\hline Trigonal bipyramid & $p_{a_{1}}(x, y, z)=(r, 0,0), p_{a_{2}}(x, y, z)=\left(-r \cdot \sin \left(30^{\circ}\right), r \cdot \sin \left(60^{\circ}\right), 0\right)$ \\
& $p_{a_{4}}(x, y, z)=(0,0, r), p_{a_{3}}(x, y, z)=\left(-r \cdot \sin \left(30^{\circ}\right),-r \cdot \sin \left(60^{\circ}\right), 0\right), p_{a_{5}}(x, y, z)=(0,0,-r)$ \\
\hline Octahedron & $p_{a_{1}}(x, y, z)=(r, 0,0), p_{a_{2}}(x, y, z)=(0, r, 0), p_{a_{3}}(x, y, z)=(r, 0,0)$ \\
& $p_{a_{4}}(x, y, z)=(0,-r, 0), p_{a_{5}}(x, y, z)=(0,0, r), p_{a_{6}}(x, y, z)=(0,0,-r)$ \\
\hline Pentagonal dipyramid & $p_{a_{1}}(x, y, z)=(r, 0,0), p_{a_{2}}(x, y, z)=\left(r \cdot \cos 72^{\circ}, r \cdot \sin 72^{\circ}, 0\right)$ \\
& $p_{a_{3}}(x, y, z)=(0,0, r), p_{a_{4}}(x, y, z)=\left(-r \cdot \cos 36^{\circ}, r \cdot \sin 36^{\circ}, 0\right)$ \\
& $p_{a_{5}}(x, y, z)=(0,0,-r), p_{a_{6}}(x, y, z)=\left(r \cdot \cos 72^{\circ},-r \cdot \sin 72^{\circ}, 0\right), p_{a_{7}}(x, y, z)=\left(-r \cdot \cos 36^{\circ},-r \cdot \sin 36^{\circ}, 0\right)$ \\
\hline Square anti-prism & $p_{a_{1}}(x, y, z)=\left(r \cdot a \frac{\sqrt{2}}{2}, 0, r \cdot \frac{h}{2}\right), p_{a_{2}}(x, y, z)=\left(0, r \cdot a \frac{\sqrt{2}}{2}, r \cdot \frac{h}{2}\right), p_{a_{3}}(x, y, z)=\left(-r \cdot a \frac{\sqrt{2}}{2}, 0, r \cdot \frac{h}{2}\right)$ \\
& $p_{a_{4}}(x, y, z)=\left(0,-r \cdot a \frac{\sqrt{2}}{2}, r \cdot \frac{h}{2}\right), p_{a_{5}}(x, y, z)=\left(r \cdot a, r \cdot a,-r \cdot \frac{h}{2}\right), p_{a_{6}}(x, y, z)=\left(-r \cdot a, r \cdot a,-r \cdot \frac{h}{2}\right)$ \\
& $p_{a_{7}}(x, y, z)=\left(-r \cdot a,-r \cdot a,-r \cdot \frac{h}{2}\right), p_{a_{8}}(x, y, z)=\left(r \cdot a,-r \cdot a,-r \cdot \frac{h}{2}\right)(h / 2 \approx 0.5237 a \approx 1.2156)$ \\
\hline
\end{tabular}

its ID and current 3-D location $((\mathrm{x}, \mathrm{y}, \mathrm{z})$ coordinates). When

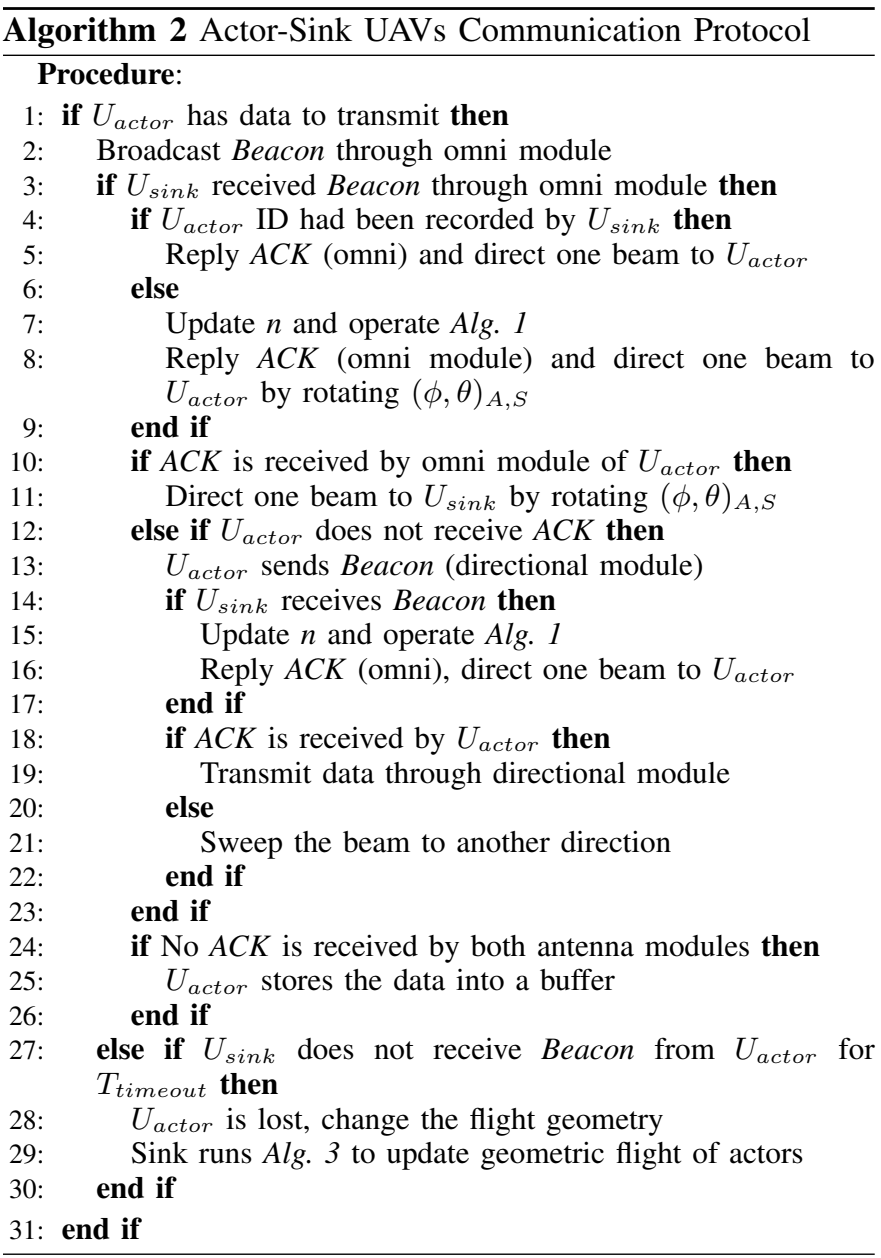

the sink receives the beacon messages, it checks the actor's connection record. If it is not found, the sink updates the number of neighbor actors and determines the VSEPR geometry by using Algorithm 1. Then the actors start changing their positions relative to the sink and create a new beamforming.

The communication links of omni module may fail occasionally due to obstacles, interference or low RSSI value caused by its orientation. We propose Algorithm 3 to make O-BESPAR antenna model adaptable to the dynamics of geometrical flight model. If the sink does not receive any beacon from an actor, which has been connected for $T_{\text {timeout }}$, it assumes that the actor has left the geometry and sends out a Position Update Message (PUM). PUM has 10 bytes and it is used to rearrange actors' positions with the purpose of maximizing network coverage.

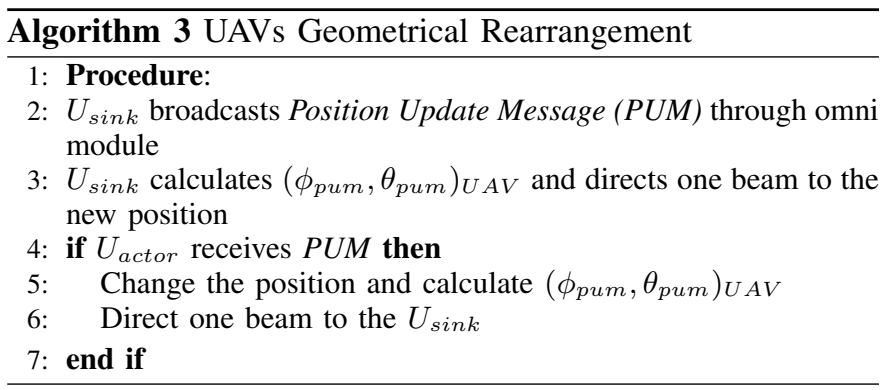

\section{Simulation Study}

The simulation study is conducted in the ns- 2 simulator. The O-BESPAR antenna model, VSEPR theory flight structures, the communication and flight control protocols are implemented and tested. The performance of packet reception ratio (PRR), actors' reorganization delay, and RSSI are evaluated to show the efficiency of the algorithm. The relationship between RSSI and coverage by two antenna models is also discussed.

\section{A. Simulation configuration}

There are 30 UAVs in the simulation, two to eight of which are actors and one of which is the sink. Zigbee which has been integrated in many off-the-shelf sensors on UAV is implemented for MAC and physical layer communications. The target area size is $1000 \mathrm{~m} \times 1000 \mathrm{~m}$. According to the specs of UAVs [20], the simulation time is set to 15 minutes and the flying speed of actor and sink is $1 \mathrm{~m} / \mathrm{s}$. The sink and actors fly with a predefined plan while maintaining the geometry. We assume the sensor UAVs fly at altitudes different from the actors and the sink. Their flying speed and movements are random and their communications cause interference to the 
UAVs in geometrical flight. Five beacons per second are sent out by the actor to search for the sink. Each UAV includes a queue of 50 packets.

O-BESPAR antenna works in $2.4 \mathrm{GHz}$ frequency band. The sink and actor UAVs have the same antenna structures. The transmission radius of omni module on each UAV is 10 meters. According to the relationship of transmission range between omni and directional modules [8], the transmission distance of the beamforming is 28 meters. For bi-directional beam sweeping, the beamwidth is configured as $60^{\circ}$. Therefore the sweeping angle of each time is $60^{\circ}$ and the beam sweeps from 0 to $180^{\circ}$.

\section{B. Simulation Results}

1) Packet transmission experiment: The PRR of two antennas are evaluated for all of the VSEPR geometries. Fig. 2 and 3 show the PRR performance of the omni and O-BESPAR antennas in different geometries (shown in Fig. 1) when the number of actors increases from two to eight. When the number of actors are lower than six, the PRR of omni antenna is between $50 \%$ and $60 \%$. The PRR of O-BESPAR antenna varied between $97 \%$ and $99 \%$. In pentagonal bipyramid and square antiprismatic geometries, the PRR of actor UAVs drops between $40 \%$ and $50 \%$ by using omni antenna. Meanwhile, OBESPAR antenna guarantees the PRR of AWSANs higher than 95\%. The packet loss of omnidirectional antenna increases as more actors transmit packets to the sink. There are two fundamental reasons for packet loss. Due to the interference from other non-actor UAVs in flight, omni antenna has much more transmission collisions than O-BESPAR antenna. In addition, the actor has varied link quality values as the RSSI is affected by antenna orientation. The PRR of actor 1 drops in square antiprismatic geometry because of the poor RSSI at that orientation. For O-BESPAR antenna, the increase of number of actors makes directional module of the sink busy. The data packet is lost if the buffer is full or the timestamp is expired.

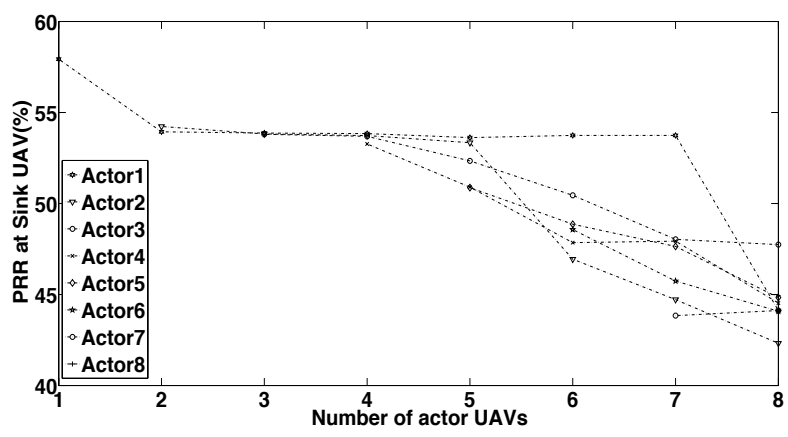

Fig. 2. PRR of Omnidirectional antenna model in different geometries.

2) Reorganization experiment: The actor-sink link is prone to failure due to flying path dynamics and interference. Then the sink repositions actors and updates the geometry for a better coverage. This process is called reorganization. In this experiment, UAV actor 1 leaves the network during the flight.

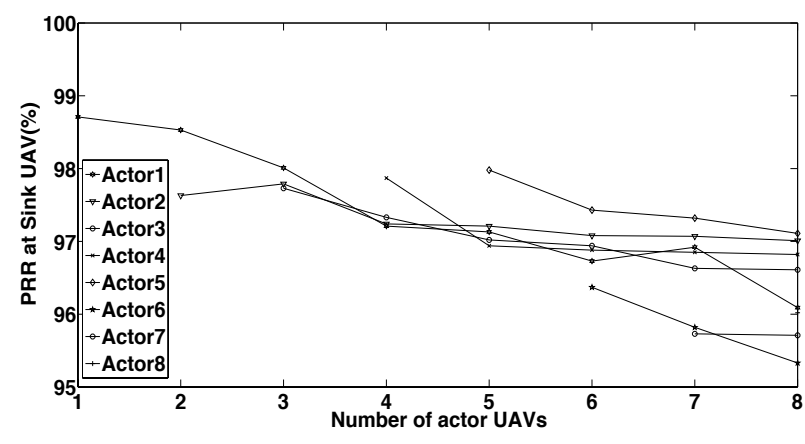

Fig. 3. PRR of O-BESPAR antenna model in different geometries.

Then, sink uses Algorithm 3 and changes the flight geometry. The positions of the actors must be updated with the PUM message for transitioning to the new geometry. The time delay is defined as the duration between the actor 1 leaving and all actors receiving PUM message. Fig. 4 presents the reorganization time delay of omni and O-BESPAR antennas in different geometries.

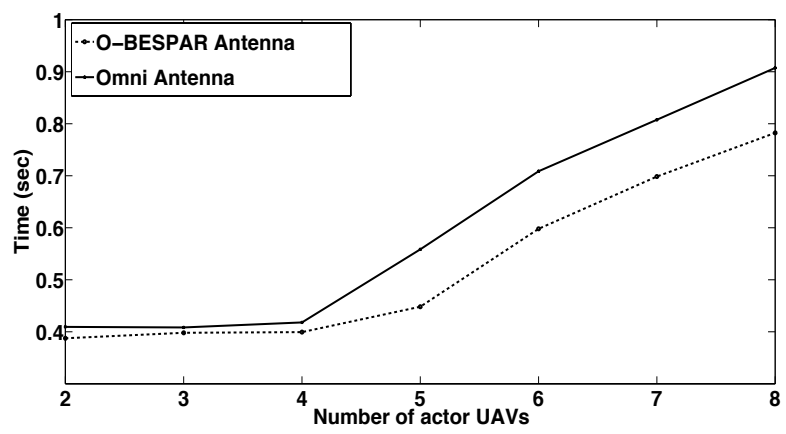

Fig. 4. Reorganization time delay of antenna models

As Fig. 4 shows, larger number of actors results in longer reorganization delay. Generally, the time delay of both antenna models is similar since O-BESPAR also uses omni antenna module to search neighboring UAVs. However, there is a time gap between two models. For omni antenna, as a result of transmission of both control and data packets, either actor or sink does not exchange beacons until the end of data transmission. It causes a long reception delay of control packets. For O-BESPAR antenna, only the beacon packets are sent through omni module, which minimizes the time delay of actor relocation.

3) Antenna orientation and coverage: As discussed in the previous section, the orientation of omni antenna module causes poor RSSI which decreases PRR at some positions. The loss of beacon messages increases reorganization time delay of UAVs. Therefore, those UAVs with poor RSSI need to fly closer to the sink in order to guarantee the RSSI. However, as distance between the actor and the sink becomes shorter, network coverage becomes smaller. As a result, there is a tradeoff between coverage and RSSI of UAVs.

Fig. 5 shows the RSSI of each actor UAV in the geometry flight. Most of actors maintain the RSSI in each geometry, 


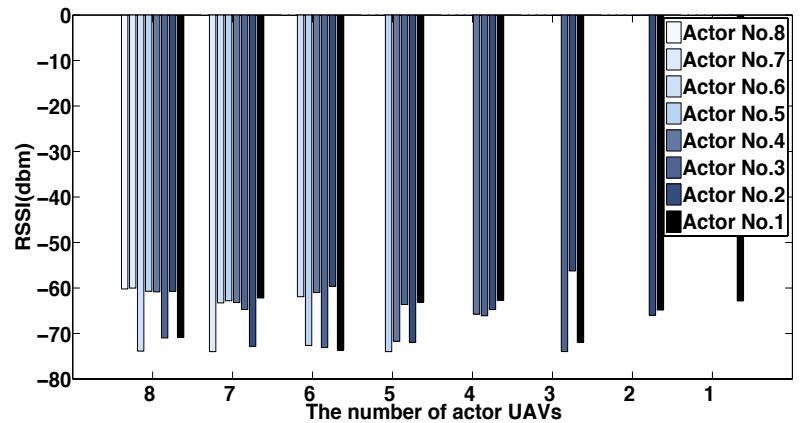

Fig. 5. RSSI values of the omni antenna in different geometry

however, some actors have higher beacon packet loss due to poor RSSI of the orientation. Based on the RSSI values in different geometries, the actor whose RSSI is smaller than the original flies closer to the sink to achieve the same RSSI.

When the actors fly closer to the sink, the coverage of network is changed. The coverage is measured by the volume covered by the network geometry in the air. Fig. 6 presents the original and updated network coverage values. Total coverage of the network is critical since when it increases, the data sensing range of the actors also increases. The network has to sacrifice at most $13 \%$ of coverage to fulfill the RSSI requirement of omni antenna. In particular, this tradeoff between RSSI and network coverage can be used as an indication of the network design, such as the requirements of delay tolerant AWSAN.

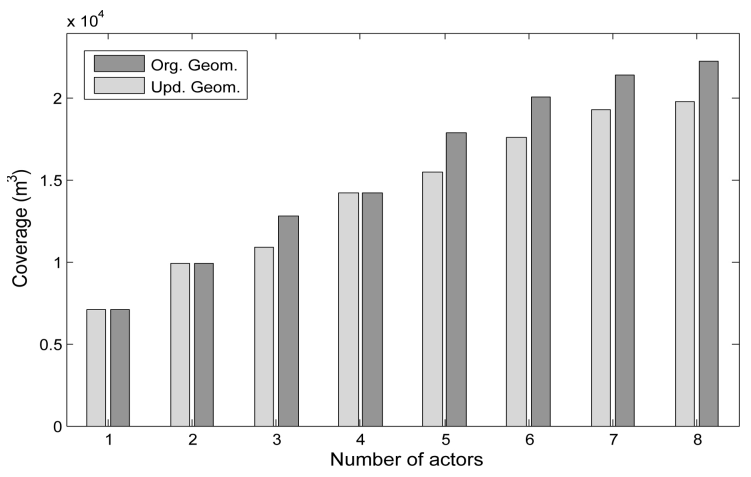

Fig. 6. The UAV coverage of each geometry flight

\section{CONClusion}

In this paper, we present a positioning approach which utilizes O-BESPAR antenna model in AWSANs. The positioning algorithm, which is based on VSEPR theory, is proved to achieve effective coverage for AWSANs. However, the dynamic positioning requirement, defective signal strength of omnidirectional antenna and unreliability of links pose challenges for AWSANs. Our algorithms take advantage of the hybrid antenna model to improve the efficiency and availability. We present extensive simulations with 3-D radio characterization to demonstrate the improvement of PRR and network reorganization delay. The variance of network coverage caused by omnidirectional antenna orientation is also discussed. As future work, we plan to build O-BESPAR antenna module and test the performance of our approach in a real 3-D testbed.

\section{REFERENCES}

[1] I. F. Akyildiz and I. H. Kasimoglu, "Wireless sensor and actor networks: research challenges," Ad Hoc Networks, vol. 2, no. 4, pp. 351-367, Oct. 2004.

[2] A. Viguria, I. Maza, and A. Ollero, "Distributed Service-Based Cooperation in Aerial/Ground Robot Teams Applied to Fire Detection and Extinguishing Missions," Advanced Robotics, vol. 24, no. 1-2, pp. 123, 2010.

[3] J. Berni, P. J. Zarco-Tejada, L. Suárez, and E. Fereres, "Thermal and Narrowband Multispectral Remote Sensing for Vegetation Monitoring From an Unmanned Aerial Vehicle," IEEE Transactions on Geoscience and Remote Sensing, vol. 47, no. 3, pp. 722-738, 2009.

[4] İ. Bekmezci, O. K. Sahingoz, and Ş. Temel, "Flying Ad-Hoc Networks (FANET): A Survey,” Ad Hoc Networks, vol. 11, no. 3, pp. 1254-1270, May 2013.

[5] S. Thrun, M. Diel, and D. Hähnel, "Scan Alignment and 3-D Surface Modeling with a Helicopter Platform," Field and Service Robotics, pp. 287-297, 2006.

[6] M. I. Akbas and D. Turgut, "APAWSAN: Actor Positioning for Aerial Wireless Sensor and Actor Networks," Proc. of IEEE Local Computer Networks Conference (LCN), pp. 567-574, Oct. 2011.

[7] M. I. Akbas, G. Solmaz and D. Turgut, "Actor Positioning Based on Molecular Geometry in Aerial Sensor Networks," Proc. of IEEE International Conference on Communications (ICC), Jun. 2012.

[8] K. Li, N. Ahmed, S. S. Kanhere, and S. Jha, "Reliable Communications in Aerial Sensor Networks by Using a Hybrid Antenna," Proc. of IEEE Local Computer Networks Conference (LCN), pp. 156-159, Oct. 2012.

[9] R. Gillespie and R. Nyholm, "Inorganic Stereochemistry," Quart. Rev. Chem. Soc., vol. 11, pp. 339-380, 1957.

[10] S. M. N. Alam and Z. J. Haas, "Coverage and Connectivity in ThreeDimensional Networks," Proc. of Annual International Conference on Mobile Computing and Networking (MobiCom), pp. 346-357, Sep. 2006.

[11] X. Bai, C. Zhang, D. Xuan, J. Teng, and W. Jia, "Low-Connectivity and Full-Coverage Three Dimensional Wireless Sensor Networks," Proc. of ACM International Symposium on Mobile Ad Hoc Networking and Computing (MobiHoc), pp. 145-154, May 2009.

[12] P. I.-S. Chiang and W.-C. Peng, "Slab Routing: Adapting TwoDimensional Geographic Routing to Three-Dimensions," Proc. of IEEE International Conference on Sensing, Communication and Networking (SECON), pp. 1-9, Jul. 2009.

[13] G. Lee, Y. Nishimura, K. Tatara, and N. Y. Chong, "Three Dimensional Deployment of Robot Swarms," Proc. of International Conference on Intelligent Robots and Systems (IROS), pp. 5073-5078, Oct. 2010.

[14] P. Li, C. Zhang, and Y. Fang, "The Capacity of Wireless Ad Hoc Networks Using Directional Antennas," IEEE Transactions on Mobile Computing, vol. 10, no. 10, pp. 1374-1387, Oct. 2011.

[15] J. Jiang, C. Lin, and Y. Hsu, "Localization with Rotatable Directional Antennas for Wireless Sensor Networks," Proc. of IEEE International Conference on Parallel Processing Workshops (ICPPW), pp. 542-548, Sep. 2010.

[16] V. Navda, A. P. Subramanian, K. Dhanasekaran, A. Timm-Giel, and S. Das, "MobiSteer: Using Steerable Beam Directional Antenna for Vehicular Network Access," Proc. of International Conference on Mobile Systems, Applications and Services (MobiSys), pp. 192-205, 2007.

[17] G. Jakllari, W. Luo, and S. V. Krishnamurthy, "An Integrated Neighbor Discovery and MAC Protocol for Ad Hoc Networks Using Directional Antennas," IEEE Transactions on Wireless Communications, vol. 6, no. 3, pp. 1114-1024, 2007.

[18] A. Alshbatat and L. Dong, "Performance Analysis of Mobile Ad Hoc Unmanned Aerial Vehicle Communication Networks with Directional Antennas," International Journal of Aerospace Engineering, 2011.

[19] N. Ahmed, S. S. Kanhere, and S. Jha, "Link Characterization for Aerial Wireless Sensor Networks," Proc. of IEEE Global Communications Conference (GLOBECOM) Workshops, pp. 1274-1279, Dec. 2011.

[20] "UAV flight time," 04 2012. [Online]. Available: http://www.mikrokopter.de/ucwiki/en/flighttime 